OPEN ACCESS

Edited by:

Francesca Turroni,

University of Parma, Italy

Reviewed by:

Xin'e Shi,

Northwest A\&F University, China Jiangchao Zhao,

University of Arkansas, United States

${ }^{*}$ Correspondence:

Koen Venema

k.venema@maastrichtuniversity.nl

Specialty section:

This article was submitted to

Microbial Symbioses,

a section of the journal

Frontiers in Microbiology

Received: 09 June 2020

Accepted: 12 August 2020

Published: 28 August 2020

Citation:

Long $C$, de Vries $S$ and Venema $K$

(2020) Differently Pre-treated

Rapeseed Meals Affect in vitro Swine

Gut Microbiota Composition.

Front. Microbiol. 11:570985.

doi: 10.3389/fmicb.2020.570985

\section{Differently Pre-treated Rapeseed Meals Affect in vitro Swine Gut Microbiota Composition}

\author{
Cheng Long ${ }^{1,2}$, Sonja de Vries $^{3}$ and Koen Venema ${ }^{1,2 *}$ \\ ' Faculty of Science and Engineering, Centre for Healthy Eating and Food Innovation, Maastricht University, Maastricht, \\ Netherlands, ${ }^{2}$ School of Nutrition and Translational Research in Metabolism (NUTRIM), Maastricht University, Maastricht, \\ Netherlands, ${ }^{3}$ Animal Nutrition Group, Wageningen University \& Research, Wageningen, Netherlands
}

The aim of the study was to investigate the effect of untreated and processed rapeseed meal (RSM) on fiber degradability by pig gut microbiota and the adaptation of the microbiota to the substrate, by using the Swine Large Intestine in vitro Model (SLIM). A standardized swine gut microbiota was fed for $48 \mathrm{~h}$ with predigested RSM which was processed enzymatically by a cellulase (CELL), two pectinases (PECT), or chemically by an alkaline (ALK) treatment. Amplicons of the V3-V4 region of the 16S rRNA gene were sequenced to evaluate the gut microbiota composition, whereas short chain fatty acids (SCFA) were measured to assess fiber degradation. Adaptive gPCA showed that CELL and ALK had larger effects on the microbiota composition than PECT1 and PECT2, and all substrates had larger effects than CON. The relative abundance of family Prevotellaceae was significantly higher in CELL treatment compared to other treatments. Regardless of the treatments (including CON), the relative abundance of Dorea, Allisonella, and FamilyXIIIUCG_001 (in the order of Clostridiales) were significantly increased after 24 h, and Parabacteroides, Mogibacterium, Intestinimonas, Oscillibacter, RuminococcaceaeUCG_009, Acidaminococcus, Sutterella, and Citrobacter were significantly higher in abundance at time point 48 compared to the earlier time points. Prevotella 9 had significant positive correlations with propionic and valeric acid, and Mogibacterium positively correlated with acetic and caproic acid. There was no significant difference in SCFA production between untreated and processed RSM. Overall, degradability in the processed RSM was not improved compared to CON. However, the significantly different microbes detected among treatments, and the bacteria considerably correlating with SCFA production might be important findings to determine strategies to shorten the fiber adaptation period of the microbiota, in order to increase feed efficiency in the animal, and particularly in pig production.

Keywords: rapeseed meal, pig gut microbiota, cellulase, pectinase, alkaline, adaptation period 


\section{INTRODUCTION}

Rapeseed meal (RSM) is not only an important alternative feed ingredient for protein-rich feeds (where mainly soybean meal is used as protein source), but RSM is also rich in nonstarch polysaccharides (NSP) (Pustjens, 2013; De Vries et al., 2014; Pustjens et al., 2014a). However, a disadvantage of RSM is that a high proportion of cell wall polysaccharides cannot be utilized by endogenous enzymes from monogastric animals, and the NSP can affect the digestion of other nutrients by physical hindrance or physiological shift in the intestine (e.g., increasing digesta viscosity) (De Vries et al., 2014). NSP can only be fermented in the swine large intestine by the gut microbiota, and research has shown that the pig gut microbiota needs an adaptation period to express its maximum enzymatic potential after a dietary change (Castillo et al., 2007). However, most of the scientific research focused on how the fibers status affected nutrient digestibility or animal performance during the adaptation period (Huang et al., 2018; Lyu et al., 2018), and few monitored how the gut microbiota changed during this period (Umu et al., 2018).

Previous studies showed that a high-fiber rapeseed diet did not results in a significant increase in SCFA content in the chyme of RSM-fed pigs after a 3-week adaptation period (Chen et al., 2018; Umu et al., 2018). However, SCFA-producing microbes, such as Dialister, Shuttleworthia, Bulleidia, Coprococcus, and Lachnospira, were detected more abundant in the colon of highfiber rapeseed pigs compared to those in the control pigs (Umu et al., 2018). This might be because the 3-week adaptation period was too short to have a considerable change in the metabolic function of SCFA-producing microbes, or more likely, the SCFA were rapidly utilized by the intestinal epithelium of the host. Adaptation duration in pigs supplemented with 19.5\% palm kernel showed that 3 weeks of adaptation is needed, and a longer adaptation time is suggested as dietary palm kernel content of the diet increases (Huang et al., 2018). Huang et al. (2018) also observed that apparent total tract digestibility of acid detergent fiber significantly increased when the adaptation time increased from 7 to 28 days (Huang et al., 2018). Another report showed that microbial cellulase activity was only observed after a 6-week adaptation period when pigs were fed with different types of dietary fiber ( $8 \%$ sugar beet pulp, or $10 \%$ wheat bran) (Castillo et al., 2007). Montoya et al. (2018) demonstrated that an optimum adaptation time also depends on the dietary level when growing pigs were fed with kiwi fruit (Montoya et al., 2018). Thus, the optimal adaptation time should be evaluated case by case.

Treatment with carbohydrases, such as cellulase and pectinase, have been reported to improve the NSP degradation after a 4-week adaptation in both boilers and in vitro (Pustjens et al., 2012; De Vries et al., 2014). Thus, in the current study, RSM (predigested) was treated independently with two kinds of pectinases (PECT1 and PECT2), one cellulase (CELL), or alkaline (ALK), and afterward the untreated and treated RSM preparations were fed to the recently developed Swine Large Intestine in vitro Model (SLIM) (Long et al., 2020) for a $48 \mathrm{~h}$ period. SLIM was established on the basis of the human, computer-controlled, dynamic TNO in vitro model of the colon, TIM-2 (Minekus et al., 1999), which accurately simulates the physiological conditions in the lumen of the human proximal colon, containing a gut microbiota of human origin. A unique design of the machine is that the model is equipped with a dialyzate system, which prevents accumulation of microbial metabolites which would lead to the inhibition or death of microbes in the model when they would accumulate. Since all metabolites (i.e., SCFA) are collected, and basically a massbalance can be made, this allows for real determination of the production of microbial metabolites such as SCFA, which is not possible in vivo.

In the current study, the effects of processed rapeseed meal on swine gut microbiota and SCFA production were investigated. We hypothesized that (1) the treatments on RSM will increase SCFA production (fiber degradability) during the fiber fermentation period compared to when the swine gut microbiota is fed with untreated RSM, and (2) the effects of processed RSM on the swine gut microbiota are different according to the treatments. The results of the current study give insight into how processed RSM affect pig gut microbiota during the fermentation period, which is important information to use to shorten the feed adaptation period and to improve feed efficiency.

\section{MATERIALS AND METHODS}

\section{Substrate Preparation}

Rapeseed meal (Brassica napus, Cargill N.V., Antwerp, Belgium; 2011) was obtained from a commercial feed mill (Agrifirm B.V., Utrecht, Netherlands). Preparation method I (predigesting RSM after carbohydrase or alkaline treatment; Figure 1): to $200 \mathrm{~g}$ of substrate $40 \mathrm{~mL} 10^{*}$ gastric electrolyte concentrate solution (GES, $310 \mathrm{~g}$ sodium chloride, $110 \mathrm{~g}$ potassium chloride, $15 \mathrm{~g}$ calcium chloride di-hydrate, and $4840 \mathrm{~g}$ ultrapure water) and $360 \mathrm{~mL}$ ultrapure water were added. The $\mathrm{pH}$ was adjusted to 5.5 and then nothing $(\mathrm{CON})$, ALK $(6 \mathrm{M} \mathrm{NaOH})$ or $10 \mathrm{~mL}$ of the following carbohydrases were added; CELL, Accellerase 1000 (Sigma-Aldrich, Missouri, United States); PECT1, Pectinex Ultra SP (Novozymes A/S, Bagsvaerd, Denmark); or PECT2, Multifect Pectinase (DuPont Industrial Biosciences, Genencor division, Rochester, NY). Enzyme preparations were incubated at $37^{\circ} \mathrm{C}$ for $2 \mathrm{~h}$, with occasional shaking (every $30 \mathrm{~min}$ ), while ALK was incubated overnight at $4^{\circ} \mathrm{C}$. Enzyme preparations were then heated at $100^{\circ} \mathrm{C}$ for $5 \mathrm{~min}$ to inactive enzymes. Afterward, for all five samples, $120 \mathrm{~mL}$ GES was added and the $\mathrm{pH}$ adjusted to 3 to continue with the gastric incubation according to the pre-digestion protocol as described elsewhere (Sáyago-Ayerdi et al., 2017). After pre-digestion, the slurry was centrifuged $\left(8.000 \mathrm{~g}\right.$, at $4^{\circ} \mathrm{C}$, for $\left.20 \mathrm{~min}\right)$ and dialysis was performed for the supernatants. For dialysis, a dialysis membrane (Sureflux, Nipro Europe Group Companies, Mechelen, Belgium) was used with a peristaltic pump to remove small digestion products and water. After reduction of the total volume to $\sim 450-500 \mathrm{~mL}$, supernatant was mixed with pellet, and freeze-dried. Method II (predigesting RSM before carbohydrase or alkaline treatment; 


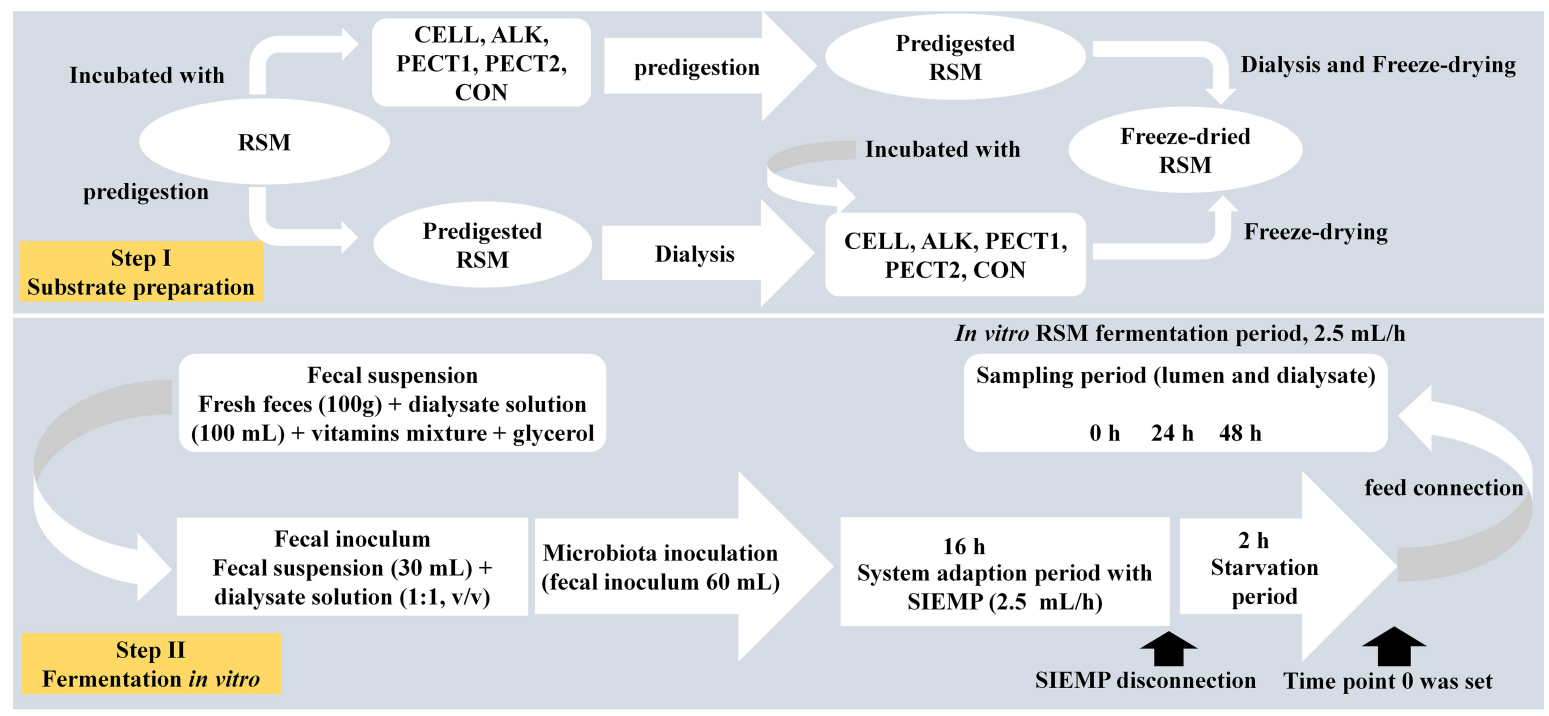

FIGURE 1 | Schematic presentation of experimental setup for fiber fermentation in the Swine Large Intestine in vitro model (SLIM).

Figure 1): four batches of $200 \mathrm{~g}$ RSM were predigested as described before (Sáyago-Ayerdi et al., 2017) and then dialyzed. Afterward, $55 \mathrm{~mL} \mathrm{10*GES} \mathrm{was} \mathrm{added,} \mathrm{and} \mathrm{pH}$ adjusted to 5.5, after which $10 \mathrm{~mL}$ of CELL, PECT1, PECT2, or ALK treatment commenced, respectively. Enzyme preparations were incubated at $37^{\circ} \mathrm{C}$ for $2 \mathrm{~h}$ with occasional shaking (every $30 \mathrm{~min}$ ), and ALK was incubated overnight at $4^{\circ} \mathrm{C}$. Afterward, enzyme preparations were heated at $100^{\circ} \mathrm{C}$ for $5 \mathrm{~min}$ to inactive enzymes, and $\mathrm{pH}$ was neutralized to $6.5-7$ with $\mathrm{HCl}$ or $\mathrm{NaOH}$, and the samples were freeze-dried.

\section{Fermentation in the Swine in vitro Large Intestinal Model (SLIM)}

The setup of SLIM was as follows: a fully computer-controlled in vitro model based on TIM-2 (Minekus et al., 1999) was used to mimic the swine large intestine (Supplementary Figure S1). The $\mathrm{pH}$ (5.9) was controlled by continuous addition of $2 \mathrm{M}$ sodium hydroxide. Standard ileal efflux medium of pigs (SIEMP) was used to simulate the materials entering the colon. The SIEMP, adapted from Gibson et al. (1988) and described in Long et al. (2020), contained the following components $(\mathrm{g} / \mathrm{L})$ : 74.6 maize starch, 9.0 xylan, 19.0 pectin, 9.0 amylopectin, 9.0 arabinogalactan, 9.0 arabinoxylan, 9.0 xyloglucan, 31.5 Tween 80 , 43.7 casein, 0.7 ox-bile, 43.7 bactopepton, $4.7 \mathrm{~K}_{2} \mathrm{HPO}_{4} .3 \mathrm{H}_{2} \mathrm{O}$, $0.009 \mathrm{FeSO}_{4} .7 \mathrm{H}_{2} \mathrm{O}, 8.4 \mathrm{NaCl}, 0.8 \mathrm{CaCl}_{2} .2 \mathrm{H}_{2} \mathrm{O}, 0.7 \mathrm{MgSO}_{4} .7 \mathrm{H}_{2} \mathrm{O}$, 0.05 bile, 0.02 hemin, and 0.3 cysteine. $\mathrm{HCl}$, plus $1.5 \mathrm{~mL}$ of a vitamin mixture containing (per liter): $1 \mathrm{mg}$ menadione, $0.5 \mathrm{mg}$ vitamin B12, $2 \mathrm{mg}$ D-biotin, $10 \mathrm{mg}$ pantothenate, $5 \mathrm{mg}$ p-aminobenzoic, $4 \mathrm{mg}$ thiamine and $5 \mathrm{mg}$ nicotinamide acid. The $\mathrm{pH}$ was adjusted to 5.9. SIEMP only contains indigestible carbohydrates and hence did not require pre-digestion. Dialysis liquid contained (per liter): $2.5 \mathrm{~g} \mathrm{~K} \mathrm{~K}_{2} \mathrm{HPO}_{4} .3 \mathrm{H}_{2} \mathrm{O}, 0.005 \mathrm{~g}$ $\mathrm{FeSO}_{4} .7 \mathrm{H}_{2} \mathrm{O}, 4.5 \mathrm{~g} \mathrm{NaCl}, 0.45 \mathrm{~g} \mathrm{CaCl}_{2} .2 \mathrm{H}_{2} \mathrm{O}, 0.05 \mathrm{~g}$ bile, $0.5 \mathrm{~g} \mathrm{MgSO}_{4} .7 \mathrm{H}_{2} \mathrm{O}$ and $0.4 \mathrm{~g}$ cysteine $\cdot \mathrm{HCl}$, plus $1 \mathrm{~mL}$ of the vitamin mixture. All medium components were purchased at Tritium Microbiology (Eindhoven, Netherlands). The pig fecal inoculum was a standardized microbiota from growing pigs (48 pens with 6 pigs/pen, Hypor Libra x Hypor Maxter, Hendrix Genetics, Boxmeer, Netherlands), consisting of pooled freshly collected feces from the floor, but only material from the top (so not toughing the floor) was selected. Since this was not an intervention study, feces collection from the floor of the pins of the pigs did not require ethical approval in accordance with local/national guidelines.

In order to create a complete anaerobic environment, SLIM with $90 \mathrm{~mL}$ dialyzate in each unit was flushed with gaseous nitrogen for at least $3 \mathrm{~h}$ before incorporating the standardized microbiota (Supplementary Figure S1). Thirty $\mathrm{mL}$ of the standardized microbiota was added to the system, making the total volume of each SLIM-unit $120 \mathrm{~mL}$. The microbiota was adapted to the model with SIEMP for $16 \mathrm{~h}$. During the adaptation phase (Figure 1) SIEMP was administered at $60 \mathrm{~mL} \mathrm{~d}$ [corresponding to $7.5 \mathrm{~g}$ carbohydrate $\mathrm{d}^{-1}$ ] (de Souza et al., 2014) through the feeding syringe (Supplementary Figure S1). At the end of the adaptation period, a 2-h starvation period was performed (Figure 1), which was used to allow all the carbohydrates within SIEMP to be fermented by the microbiota and after this time point 0 was set. Afterward, the 'fiber adaptation' period $(48 \mathrm{~h})$ was performed, in which the microbiota was allowed to adapt to the test products (CON, CELL, PECT1, PECT2 and ALK). During this stage, carbohydrates in SIEMP were replaced with 7.5 grams of (treated) RSM which were added continuously in the model at a rate of $2.5 \mathrm{~mL} / \mathrm{h}$ for $48 \mathrm{~h}$ (Figure 1), corresponding to $7.5 \mathrm{~g}$ carbohydrate $\mathrm{d}^{-1}$.

\section{Sample Collection}

Samples $(n=2)$ from lumen and spent dialyzate were collected at time point 0,24 , and $48 \mathrm{~h}(\mathrm{t} 0, \mathrm{t} 24$, and $\mathrm{t} 48)$. They were 
snap-frozen in liquid nitrogen and stored until analyses. Lumen samples were used to analyze microbiota composition, and both lumen and dialysis samples were analyzed for short chain fatty acid (SCFA) concentrations.

\section{Sequencing of V3-V4 Region of the $16 \mathrm{~S}$ rRNA Gene}

Microbial DNA extraction and sequencing of the V3-V4 region of the $16 \mathrm{~S}$ rRNA gene were performed by BaseClear B.V. (Leiden, Netherlands). Briefly, genomic DNA extraction was performed using the Quick-DNA ${ }^{\mathrm{TM}}$ Fecal/Soil Microbe Miniprep Kit (Zymo Research, CA, United States) according to the manufacturer's instructions. Barcoded amplicons from the V3-V4 region of $16 \mathrm{~S}$ rRNA genes were generated using a 2-step PCR. 10-25 ng genomic DNA was used as template for the first PCR with a total volume of $50 \mu \mathrm{L}$ using the 341F ( $5^{\prime}$-CCTACGGGNGGCWGCAG-3') and the 785R (5'-GACTACHVGGGTATCTAATCC-3') primers (Klindworth et al., 2013) appended with Illumina adaptor sequences. PCR products were purified (QIAquick PCR Purification Kit, Venlo, Netherlands) and the size of the PCR products were checked on a Fragment analyzer (Advanced Analytical, Ankeny, United States) and quantified by fluorometric analysis. Purified PCR products were used for the 2 nd PCR in combination with sample-specific barcoded primers (Nextera XT index kit, Illumina, CA, United States). Subsequently, PCR products were purified, checked on a Fragment analyzer and quantified, followed by multiplexing, clustering, and sequencing on an Illumina MiSeq with the paired-end (2x) 300 bp protocol and indexing. The sequencing run was analyzed with the Illumina CASAVA pipeline (v1.8.3) and demultiplexed based on sample-specific barcodes. Raw sequencing data was submitted to the European Nucleotide Archive under the accession number: PRJEB38485.

\section{Bioinformatics Analysis}

The demultiplexed raw sequences obtained from BaseClear were processed using QIIME2 pipeline (Bolyen et al., 2019). In short, reads were imported, quality filtered and dereplicated with q2-dada2 (Callahan et al., 2016), after which dada2 was performed with paired-end reads and truncations parameters as follows:the first 10 base pairs were trimmed off and at position 280 base pairs the fragment was truncated in forward reads, and at position 240 base pairs for the reverse reads. The processed sequences were used for all the downstream analyses. Alpha-diversity (Shannon index) and beta diversity [weighted and unweighted UniFrac; (Lozupone and Knight, 2005; Lozupone et al., 2007)] were analyzed by the q2-phylogeny plugin (https://github.com/qiime2/q2diversity).

\section{Adaptive Generalized PCA, gPCA}

Adaptive gPCA (Fukuyama, 2017; Fukuyama et al., 2017) was performed on the ASV-tables generated in QIIME. This is a relatively new method which can obtain a lowdimensional representation of the samples in which the axes are interpretable at a fine phylogenetic scale, which can be used to compare the dissimilarities between samples more accurately than that of UniFrac. The mathematical algorithm for gPCA can be found in the literature (Fukuyama, 2017). R packages biomformat, yaml, Biostrings, phyloseq, Hmisc, qiiime2R, vegan, ggplot2, and adaptiveGPCA were used in this analysis.

\section{Phylogenetic Investigation of Communities by Reconstruction of Unobserved States, PICRUSt2}

PICRUSt2 software (Douglas et al., 2019) was used to predict microbial functional abundances based on marker gene sequences. KEGG database was used to predict the results.

\section{Chemical Analyses}

\section{Short-Chain Fatty Acids Analyses}

Samples from lumen and dialyzate were analyzed by Brightlabs (Venlo, Netherlands) for determination of concentrations of SCFA (including the middle chain fatty acids valeric and caproic acid). Ion exclusion chromatography (IEC) was applied on an 883 Ion Chromatograph (IC; Metrohm, Herisau, Switzerland), using a Transgenomic IC Sep ICE-ION-300 column $(30 \mathrm{~cm}$ length, $7.8 \mathrm{~mm}$ diameter and $7 \mu \mathrm{m}$ particles) and a MetroSep RP2 Guard. The mobile phase consists of $1.5 \mathrm{mM}$ aqueous sulfuric acid. A column flow rate of $0.4 \mathrm{ml} \mathrm{min}^{-1}$ was used. The temperature of the column was $65^{\circ} \mathrm{C}$. The organic acids were detected using suppressed conductivity detection. Samples were centrifuged $(14,000 \mathrm{rpm}, 10 \mathrm{~min})$, and the clear supernatant was filtered through a $0.45 \mu \mathrm{m}$ PFTE filter and diluted with mobile phase (for lumen 1:5, for dialyzate 1:2). Ten microliters were loaded on the column by an autosampler 730 (Metrohm). Molecules were eluted according to their pKa.

\section{Statistical Methods}

Kruskal-Wallis Rank Sum Test was applied to compare alpha diversities (Shannon index) among different RSM treatments and time points, and Wilcoxon Rank Sum Test was used for pairwise comparison in $\mathrm{R}$ version 3.5.3 (https://www.r-project. org/). Bonferroni was used to correct $P$-values. Permutational multivariate analysis of variance [PERMANOVA; (Anderson, 2005)] was performed to test the significance of beta diversity (weighted and unweighted UniFrac) between non-processed and processed RSM in QIIME2. The results were visualized in R. Linear discriminant analysis effect size [LEfSe] (Segata et al., 2011) was used to find biomarkers between groups using relative abundances from the feature tables generated in QIIME at genus level.

Pearson correlations between continuous meta-variables (SCFA production) and taxonomic variables were calculated and visualized in R. Parameters were set as follows: Missing values for meta-variables were handled as NO imputation (replacing missing data with substituted); zeros were kept for the calculation of correlation; a minimum relative abundance of $0.1 \%$ was considered for calculation; a minimum of 4 pairs of observations were required for calculation of correlations. 
Kruskal-Wallis Rank Sum Test was conducted to compare SCFA production among CON, ALK, PECT1, PECT2, and CELL in the built-in $\mathrm{R}$ package.

\section{RESULTS}

\section{RSM With Different Treatment Methods Differentially Changed the Microbial Phylogenetical Relationship During the Fiber Fermentation Period}

There were $26839 \pm 4458$ (ALK), $26973 \pm 5187$ (CELL), $26243 \pm 4871$ (PECT1), $29597 \pm 4026$ (PECT2), and $52927 \pm 4055$ (CON) raw sequences obtained after Illumina Miseq sequencing. Filtered reads were $22612 \pm 4162$, $22862 \pm 440822612 \pm 4162,23895 \pm 2308$, and $40092 \pm 4147$ sequences in ALK, CELL, PECT1, PECT2, and CON, respectively. And finally $14106 \pm 2470,14665 \pm 3831,13265 \pm 2686$, $15332 \pm 1814$, and $21551 \pm 2348$ non-chimeric sequences were obtained for ALK, CELL, PECT1, PECT2, and CON, respectively, after quality control. Finally, 1261 ASVs were detected.

There were no significant difference in $\alpha$-diversity (Shannon Index, Ace, Chaol and Simpson) between non-processed RSM (CON) and processed RSM (ALK, PECT1, PECT2, and CELL) (Figure 2A and Supplementary S2). Shannon Index, Ace and Chaol were significantly decreased during the fiber fermentation period (Figure 2B and Supplementary S2), significant at $72 \mathrm{~h}$. Unweighted UniFrac shows that $\mathrm{CON}$ had a significant different $(P=0.02)$ microbial community compared to ALK, PECT1, PECT2, and CELL while there were no significant difference $(P=0.55)$ between non-processed and processed RSM in terms of weighted UniFrac (Supplementary Tables S1, S2).
The biplot of unweighted UniFrac (Supplementary Figure S3) showed that genus Prevotella 9 was mainly associated with t0, while Ruminococcaceae gauvreauii group, Eubacterium nodatum group, Succiniclasticum, Mogibacterium, Olsenella, Prevotellaceae NK3B31 group Bacteroidles S24-7 group Rikenellaceae RC9 gut group, and Ruminococcaceae UCG-002 were associated with t24 and t48. Afterward, adaptive gPCA (Fukuyama et al., 2017) was used to identify the dissimilarities among samples. The results show that the starting points ( $\mathrm{t} 0$ ) of all the treatments were approximately located in the same spot of the principle plane, and the later time points lay along the first adaptive gPCA axis (Figure 3A). After $48 \mathrm{~h}$ adaptation, the microbiota composition changed considerably in the processed RSM groups compared to the start time points, whereas $\mathrm{CON}$ only showed a slight difference between 448 and t0 (Figure 3B).

Alpha-diversity (Shannon index) and $\beta$-diversity (weighted and unweighted UniFrac) showed that there were no significant differences between microbiota fed with modified RSM before or after dialysis (data not shown). These indicated that predigesting before or after modifying RSM by ALK or enzymes did not differentially affect microbiome composition. Our previous study showed that predigesting before or after processing RSM had little effect on their monosaccharide constitution (Long et al. manuscript2 submitted).

\section{Fermentation Time Had Larger Effect on the Microbiota Composition During the Fiber Fermentation Period Than the Treatments on RSM Did}

Figure 4A and Supplementary Figure S4A show at the phylumlevel that the relative abundances of Bacteroidetes in response to ALK, CON, PECT1, PECT2, and CELL were quite similar to
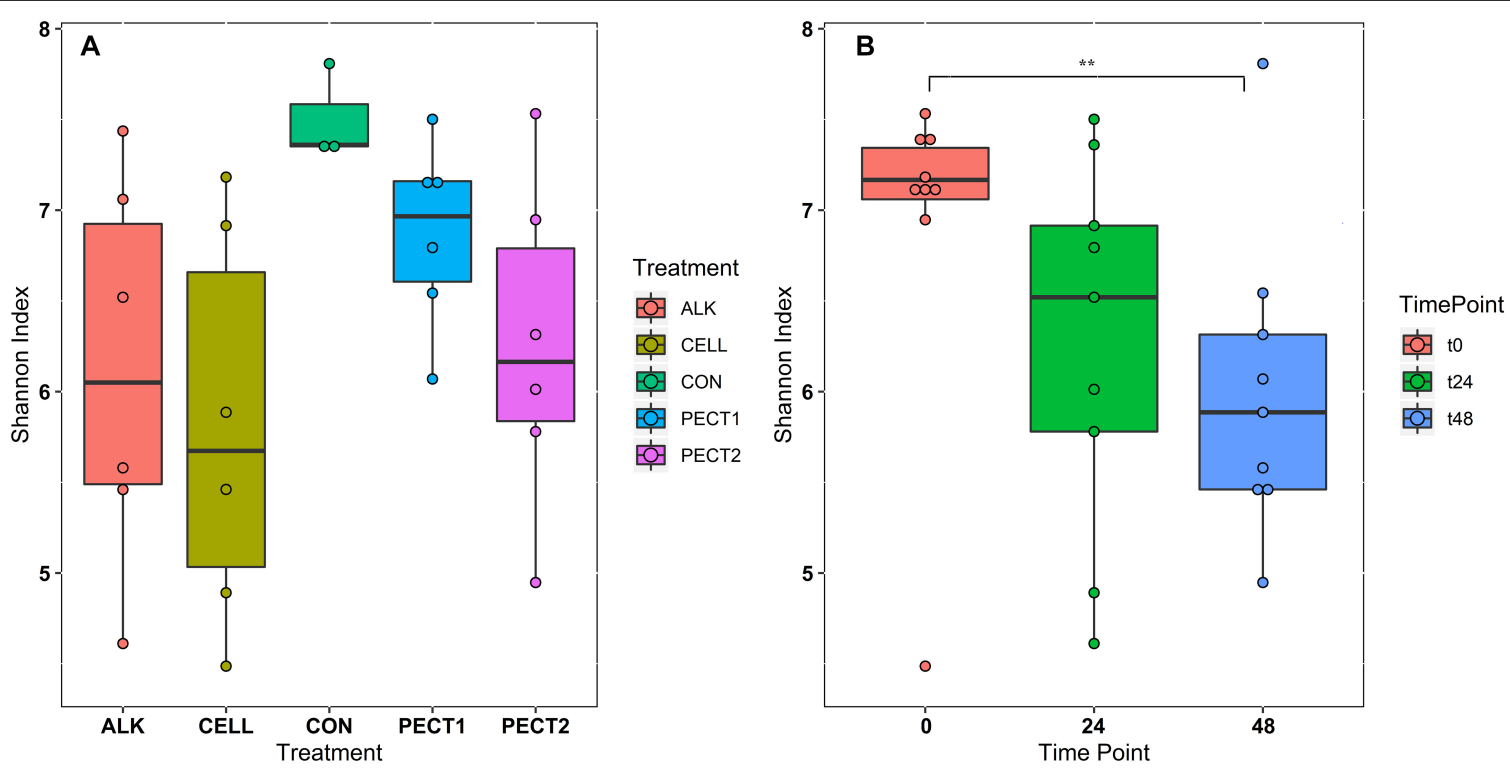

FIGURE 2 | Community $\alpha$-diversity represented by Shannon index from each treatment (A) and time point (B). The treatments are non-processed RSM (CON) and RSM processed by Accellerase 1000 (CELL), Pectinex Ultra SP (PECT1), Multifect Pectinase (PECT2), or 6 M NaOH (ALK). 

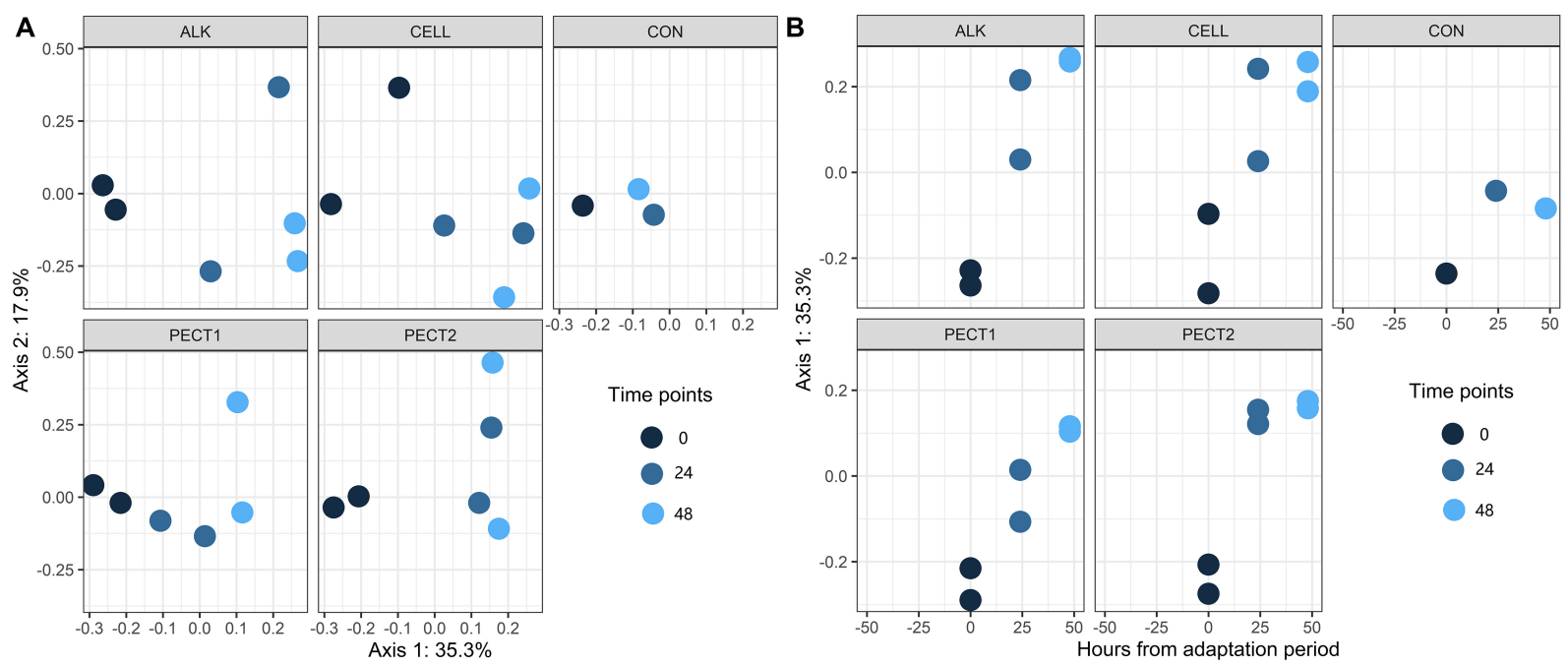

FIGURE 3 | Two views of results from adaptive gPCA. (A) shows the sample scores from gPCA plotted on the first two axes. (B) sample scores have been centered by treatments so as to better show the within-treatment variation, and the centered scores along the first axis are displayed over time.

each other at t0, whereas they continuously deceased with ALK, PECT1, and CELL feeding at both $\mathrm{t} 24$ and $\mathrm{t} 48$ compared to $\mathrm{t} 0$, while feeding CON and PECT2 decreased the relative abundances of Bacteroidetes at $\mathrm{t} 24$, which slightly increased again at $\mathrm{t} 48$. The relative abundance of Firmicutes in ALK, CON, PECT1, and PECT2 was quite stable during the fiber fermentation period, while it continuously increased in CELL. The relative abundances of Actinobacteria in ALK, PECT1, and PECT2 increased, whereas those of CON and CELL were stable during the fiber fermentation period. ALK decreased the relative abundance of Proteobacteria, while the others treatments increased this phylum during the fiber fermentation period.

Figure 4B and Supplementary Figure S4B show that the most abundant microbes at order level were: Bacteroidales, Clostridiales, Coriobacteriales, and Selenomonadales. The relative abundances of Bacteroidales in response to ALK, CON, PECT1, PECT2, and CELL were quite similar to each other at t0 (as expected), whereas they continuously deceased with ALK, PECT2, and CELL feeding at both $\mathrm{t} 24$ and $\mathrm{t} 48$ compared to $\mathrm{t} 0$, while feeding $\mathrm{CON}$ and PECT2 decreased the relative abundances of Bacteroidetes at $\mathrm{t} 24$, which slightly increased again at $\mathrm{t} 48$. The relative abundance of Clostridiales in ALK, CON, and CELL increased, and that of PECT1 was stable during the fiber fermentation period, whereas Clostridiales continuously deceased with PECT2 feeding at both $\mathrm{t} 24$ and $\mathrm{t} 48$ compared to t0. ALK, PECT1, and PECT2 increased the relative abundance of Coriobacteriales, while that of CON decreased and that of CELL was stable. The relative abundance of Selenomonadales in CON increased, while those of ALK, PECT1, PECT2, and CELL were quite stable.

Figure 4C and Supplementary Figure S4C show that the different substrates had an effect on relative abundance at the family level, and the top six most abundant families are summarized below. ALK, PECT2, and CELL feeding decreased the relative abundance of Prevotellaceae, Ruminococcaceae, and Ranunculaceae. Lachnospiraceae was stable, while Atopobiaceae and Acidaminococcus increased upon feeding ALK. Lachnospiraceae decreased, Atopobiaceae increased, and Acidaminococcus was stable upon feeding PECT2. Lachnospiraceae increased, Atopobiaceae was stable, and Acidaminococcus decreased with CELL treatment. CON and PECT1 feeding decreased the relative abundance of Prevotellaceae, Lachnospiraceae, and Ranunculaceae, and stabilized Ruminococcaceae and Acidaminococcus. The numbers of Atopobiaceae decreased with CON, while it increased with PECT1.

Linear discriminant analysis effect size [LEfSe] (Segata et al., 2011) was used to find the significantly different taxonomic ASVs between treatments (Figure $\mathbf{5 A}$ and Supplementary Figure S5A) and during the fiber fermentation period (Figure 5B and Supplementary Figure S5B). The relative abundance of Family Prevotellaceae was significantly higher in CELL treatment compared to CON. The relative abundances of ten genera were significant higher in CON compared to CELL: Prevotella 9, Kurthia, Christensenellaceae R_7 group, Lachnoclostridium, Anaerotruncus, Ruminococcaceae NK4A214 group, Ruminococcaceae UCG_002, and Ruminococcaceae UCG_003, Ruminococcaceae UCG_005, and Ruminococcaceae UCG_010. There were no significantly different microbes in PECT1 and PECT2 treatments.

More significantly different microbes were detected among the different time points (Figure 5B and Supplementary Figure S5B). At genus level, the relative abundance of three microbes, Dorea, Allisonella, and FamilyXIIIUCG_001 (in the order of Clostridiales), were significantly increased after $24 \mathrm{~h}$ adaptation. The relative abundance of eight microbes were significantly higher in $t 48$ compared to the earlier time points. These were Parabacteroides, Mogibacterium, Intestinimonas, Oscillibacter, RuminococcaceaeUCG_009, Acidaminococcus, Sutterella, and Citrobacter. Figure 5B 

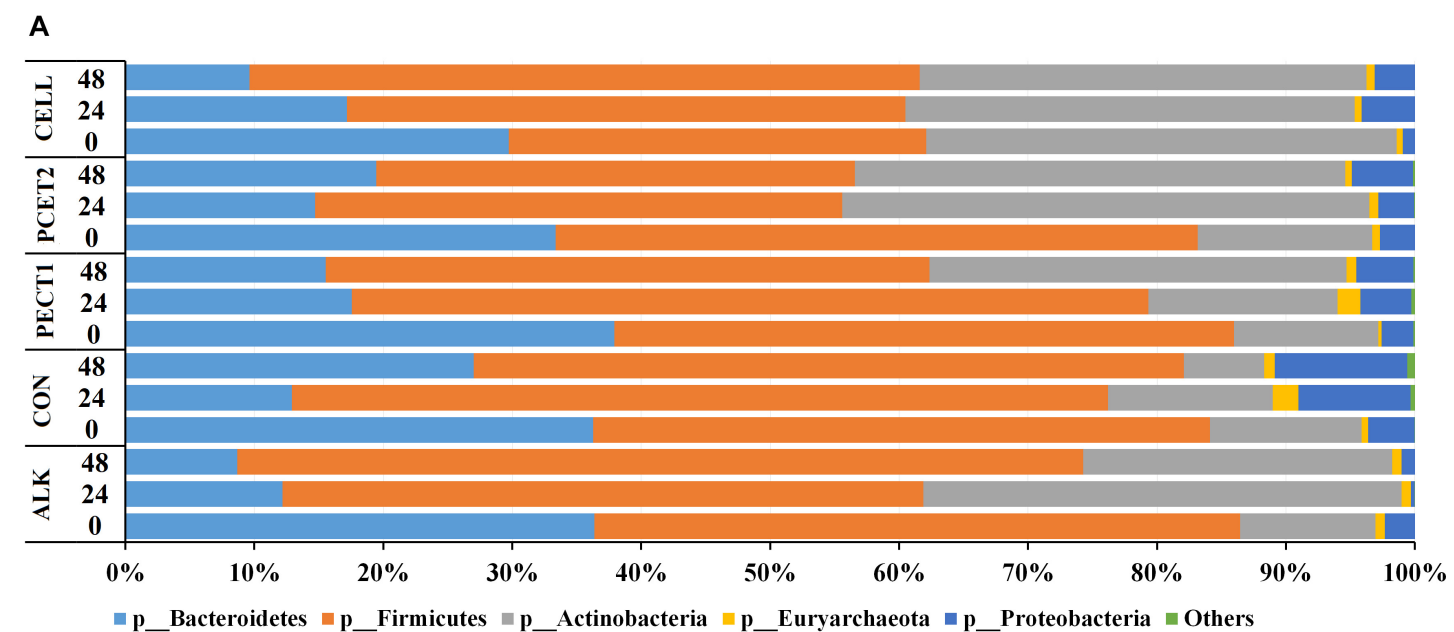

B

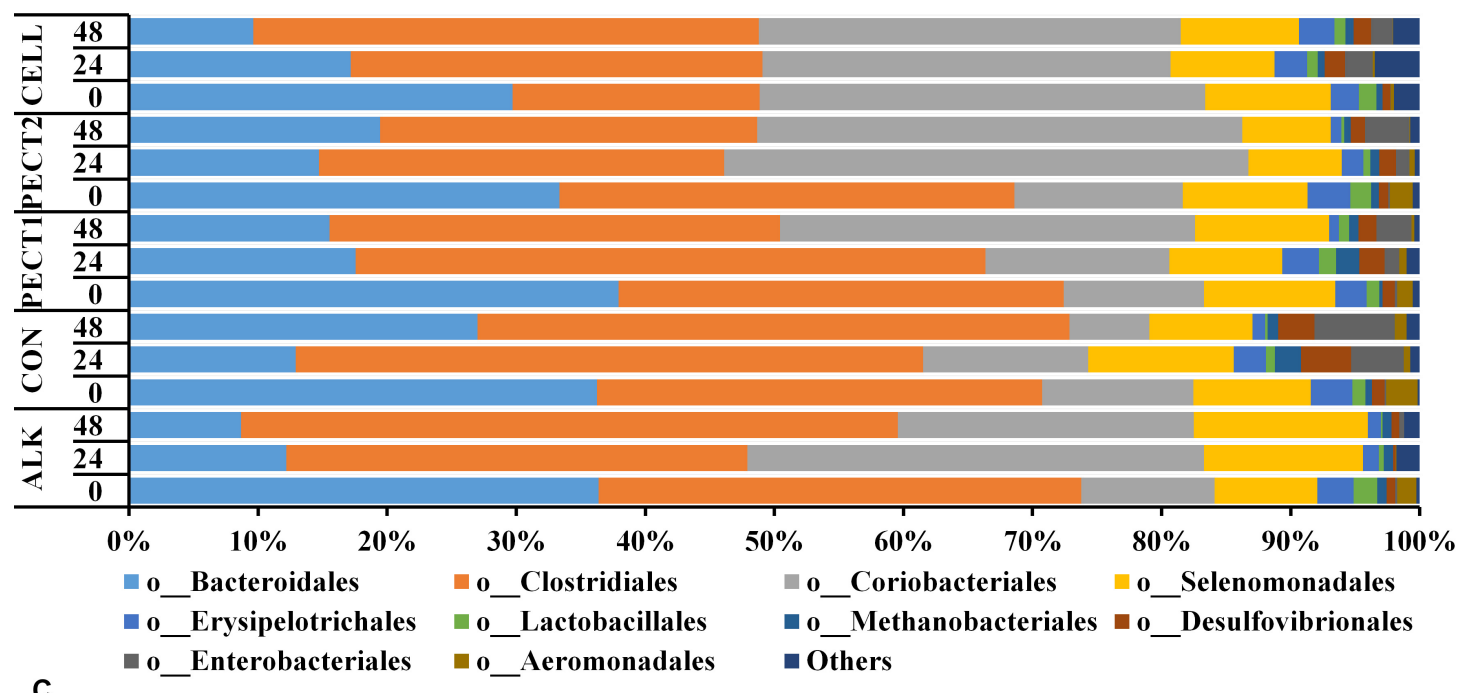

C

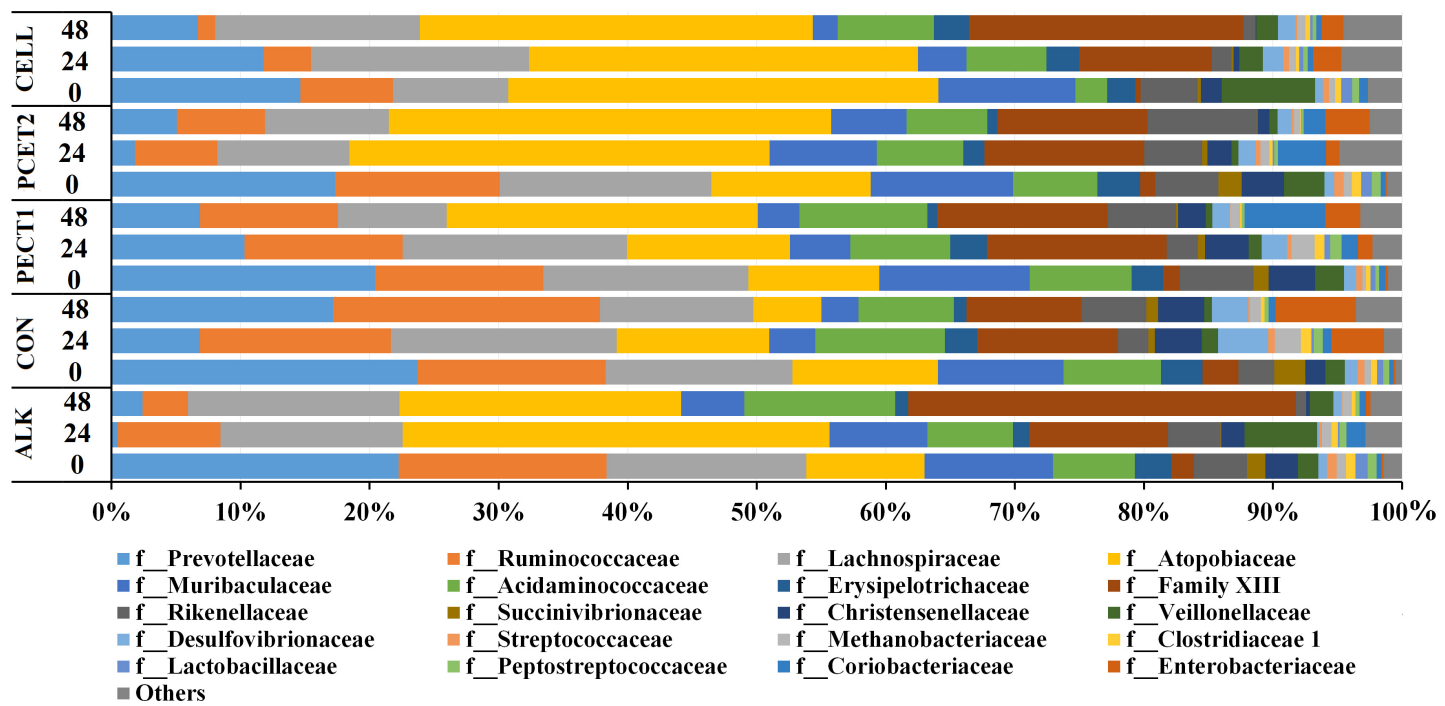

FIGURE 4 | Relative abundances of microbial phyla (A), orders (B), and families (C) in pig microbiomes fed with non-processed RSM (CON) or RSM processed by Accellerase 1000 (CELL), Pectinex Ultra SP (PECT1), Multifect Pectinase (PECT2), or $6 \mathrm{M} \mathrm{NaOH}$ (ALK) at different time points (0, 24, and 48 h). 

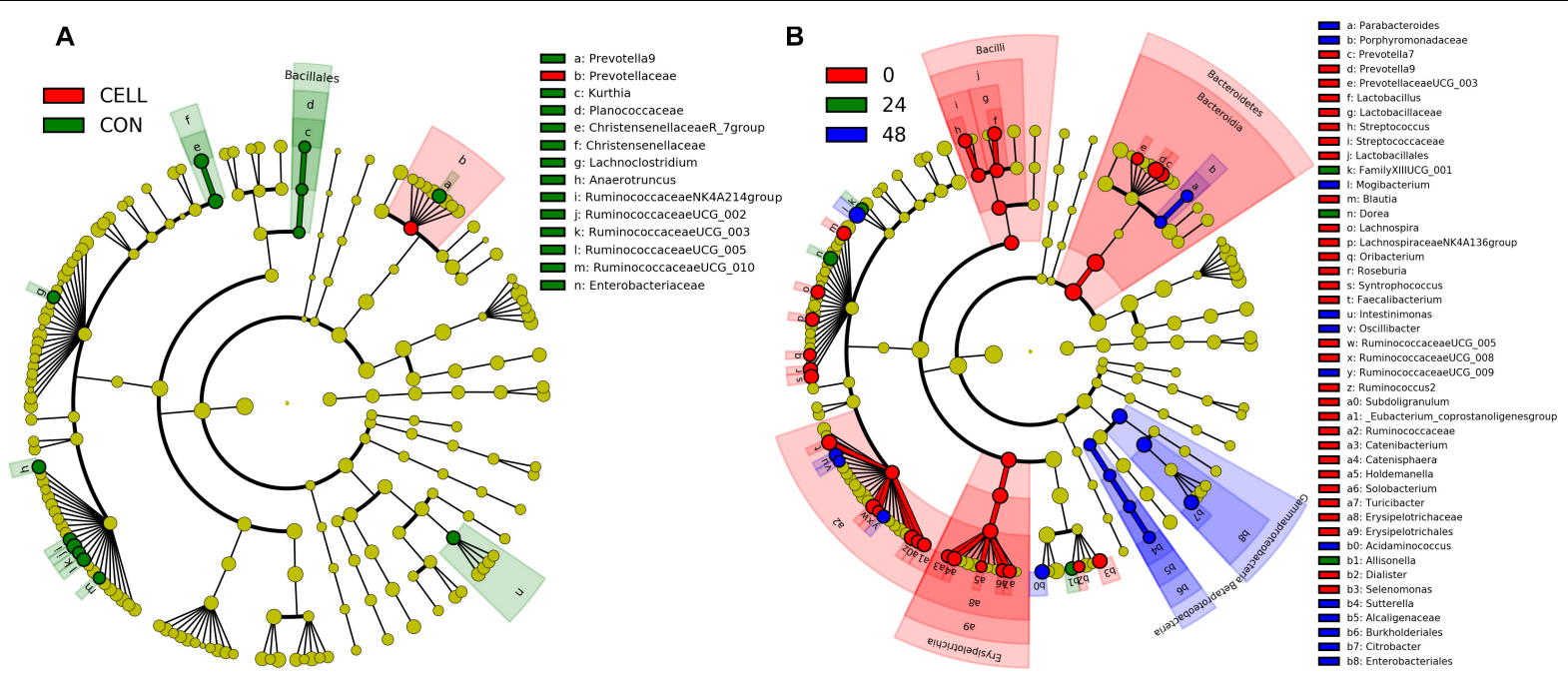

FIGURE 5 | LEfSe results of pig microbiota fed with (A) CELL and CON, and (B) at time point 0, 24, and $48 \mathrm{~h}$. Differences are represented by the color of the substrates (red, green, and blue indicating CELL and time point $0 \mathrm{~h}, \mathrm{CON}$ and time point $24 \mathrm{~h}$, and time point $48 \mathrm{~h}$, for panels $(\mathbf{A}, \mathbf{B})$, respectively).
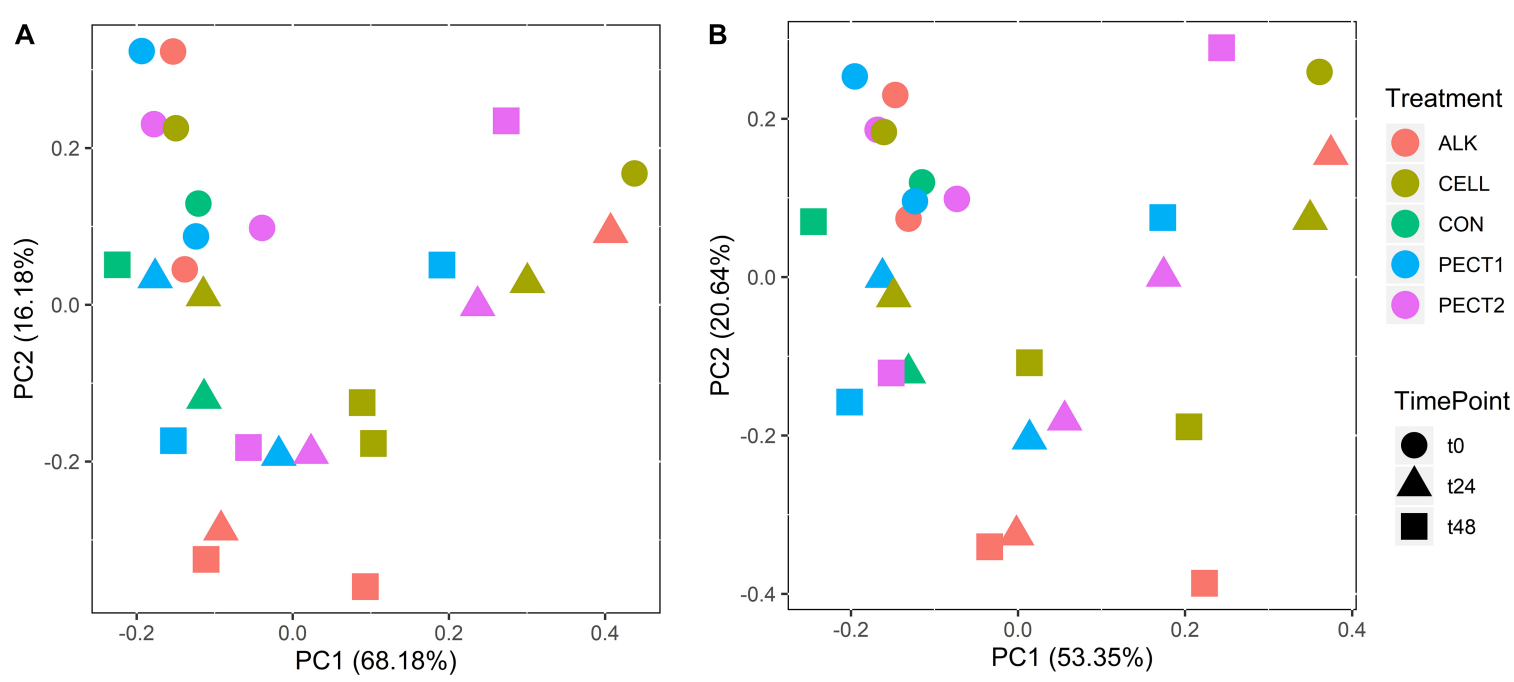

FIGURE 6 | PCA plots of the relative abundances of Enzyme Classification numbers (A) and pathways (B) from microbiota fed with CELL, CON, PECT1, PECT2, and ALK at different time points $(0,24$, and $48 \mathrm{~h})$.

shows that the relative abundance of 24 genera (linked to t0) significantly decreased after the fiber adaptation period (t24 and t48).

\section{Functional Metagenomic Profiles Changed After the Fiber Fermentation Period}

The relative abundances of PICRUSt2-predicted Enzyme Classification numbers and pathways were used to create PCA plots (Figure 6). Figure 6 shows that samples from t0 clustered together and clearly separated with samples from $\mathrm{t} 24$ and $\mathrm{t} 48$. However, there was no clearly separation $(P>0.05)$ among different treatments (Figure 6).
In this study we focused on carbohydrate metabolism related microbial functions. Figure 7 shows that 8 pathways were significantly increased after the fiber adaptation period, and they were D-galactarate degradation I, D-glucarate degradation I, glyoxylate cycle, pyruvate fermentation to acetone, TCA cycle IV (2-oxoglutarate decarboxylase), superpathway of "D-glucarate and D-galactarate degradation", superpathway of "glycolysis, pyruvate dehydrogenase, TCA, and glyoxylate bypass", and superpathway of "glyoxylate bypass and TCA". On the other hand, 4 pathways were significantly decreased after the fiber adaptation period, which were D-galacturonate degradation I, lactose and galactose degradation I, mannan degradation, and pyruvate fermentation to propanoate I. Additional significant increased other pathways and all enzymes are displayed in 

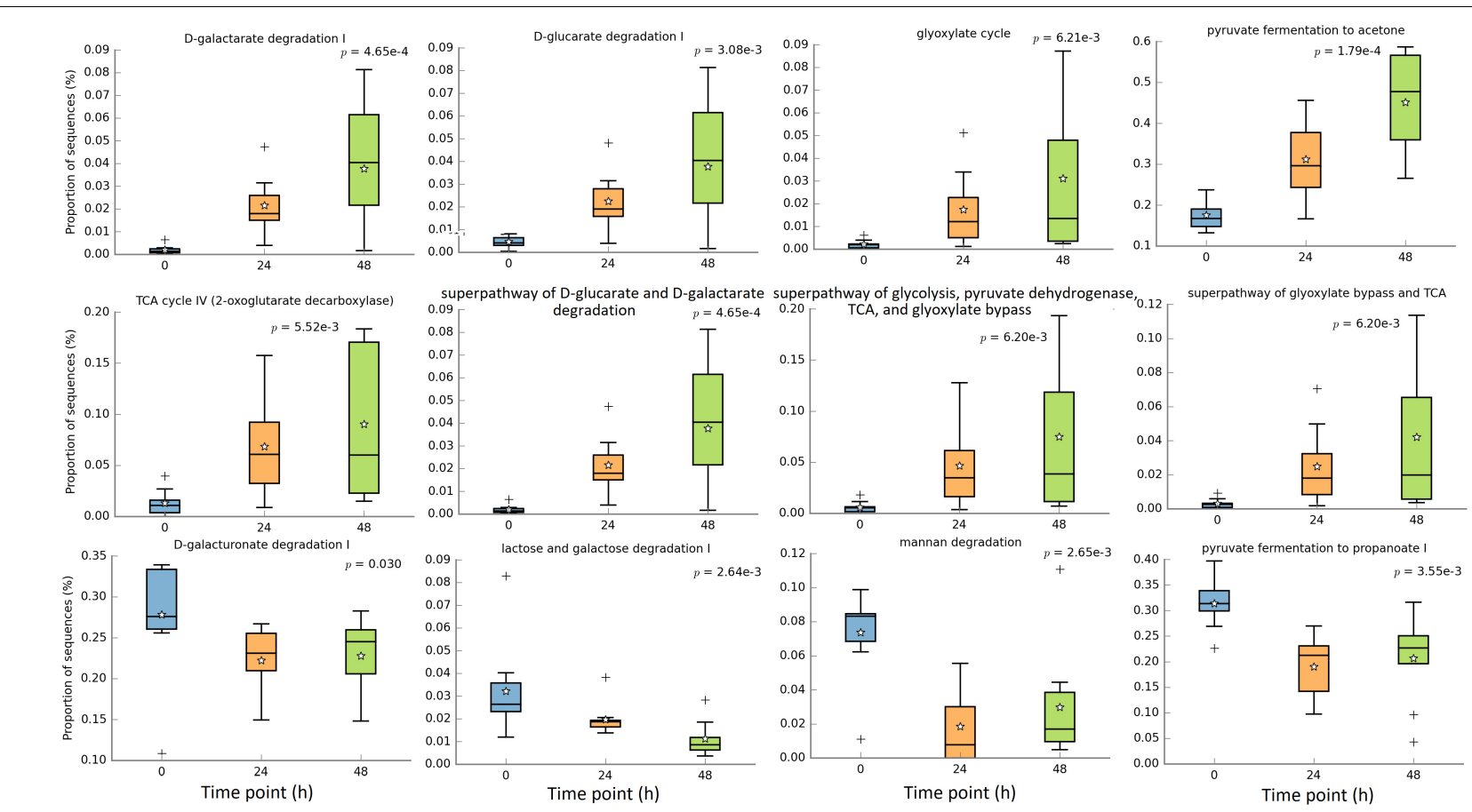

FIGURE 7 | Significantly different metagenomic functions in relative abundance among different time points.

Supplementary Tables S3, S4, respectively. There were no significantly different pathways between treatments and CON.

\section{The Cumulative Amount of SCFA Continuously Increased During the Fiber Fermentation Period}

Figure $8 \mathrm{~A}$ shows the average cumulative amount of total, individual SCFA (acetic, propionic, butyric, and including the middle chain fatty acids valeric and caproic acid) at t24 and t48. When mentioning total SCFA or SCFA production, the middle chain fatty acids valeric and caproic acid, were included in the current context. Production of total and individual SCFA constantly increased after 24 and $48 \mathrm{~h}$ of fermentation. As generally observed, more acetic acid was produced compared to the amount of propionic and butyric acid, which were similar to each other. There were no significant difference between the first $24 \mathrm{~h}$ (t24) and second $24 \mathrm{~h}$ (t48) in SCFA production, except for propionic acid production, where the amount of propionic acid production at $\mathrm{t} 24$ was significant higher than that at $448(P<0.05)$, independent of substrate (Figure $8 \mathbf{B})$. The first $24 \mathrm{~h}$ of fiber adaptation resulted in an increase of 7.0, $7.4,7.5,6.3$, and $6.6 \mathrm{mmol}$ total SCFA/g substrate in response to ALK, CELL, CON, PECT1, and PECT2, respectively. This slightly increased during the second $24 \mathrm{~h}$ of fermentation, to $7.1,8.2,7.9$, and $7.0 \mathrm{mmol}$ total SCFA/g substrate in response to ALK, CELL, CON, and PECT1 feeding, respectively. Only for PECT2 the SCFA production slightly decreased during the second $24 \mathrm{~h}$ of fermentation compared to the first $24 \mathrm{~h}(5.7 \mathrm{mmol}$ SCFA/g substrate).
There were no significant differences in SCFA production between unprocessed and processed RSM during the fiber fermentation period.

Correlations between the relative abundance at genus level and SCFA production were evaluated (Figure 8C). Prevotella $9\left(P_{\text {propionicacid }}<0.01, P_{\text {valericacid }}<0.01\right)$ and uncultured Porphyromonadaceae bacterium $\left(P_{\text {propionicacid }}=0.03\right.$, $\left.P_{\text {valericacid }}<0.01\right)$ had significant positive correlations with both propionic and valeric acid, while Mogibacterium $\left(P_{\text {propionicacid }}<0.01, P_{\text {valericacid }}<0.01\right)$ and [Eubacterium] nodatum group $\left(P_{\text {propionicacid }}=0.02, P_{\text {valericacid }}<0.01\right)$ had negative correlation with these acids. Caproic acid significantly negatively correlated with Prevotella $9(P<0.01)$, Ruminococcus $2(P=0.03)$, uncultured Porphyromonadaceae bacterium $(P=0.01)$ and Catenisphaera $(P=0.04)$, whereas Mogibacterium $\left(P_{\text {aceticacid }}=0.01, P_{\text {caproicacid }}=0.03\right)$ and [Eubacterium $]$ nodatum group $\left(P_{\text {aceticacid }}=0.01, P_{\text {caproicacid }}=0.03\right)$ positively correlated with acetic and caproic acid. An unclassified genus from Prevotellaceae $(P<0.01)$ had positive correlation with valeric acid, while Acetitomaculum $(P<0.01)$ negatively correlated with it. Subdoligranulum significantly negatively correlated with acetic $(P<0.01)$ and caproic acid $(P=0.02)$, while it had positive correlation with propionic acid $(P=0.04)$.

\section{DISCUSSION}

The pig gut microbiota needs an adaptation period to express its maximum enzymatic potential after a change of diet 

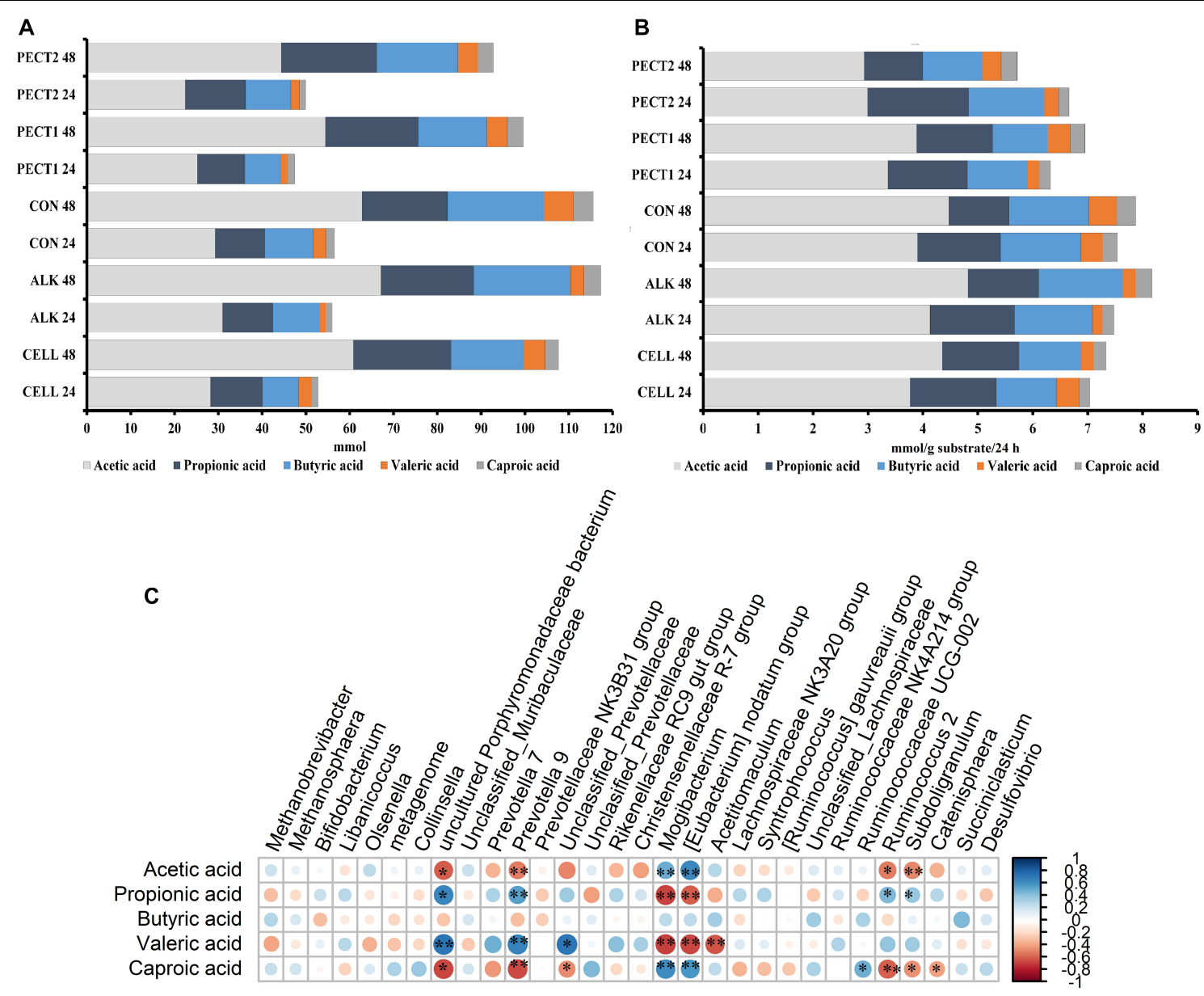

FIGURE 8 | Average cumulative short chain fatty acid (SCFA) production in mmol (A) and SCFA increase in mmol/g substrate/24 h (B) for non-processed RSM (CON) and RSM processed by Accellerase 1000 (CELL), Pectinex Ultra SP (PECT1), Multifect Pectinase (PECT2), or 6 M NaOH (ALK). The values at time point 0 were artificially set to 0 to allow determination of cumulative production. (C) correlation between SCFA production and core genera, ${ }^{\star} P<0.05$; blue: positive correlation; red: negative correlation. Core genera: the table was normalized via division by the sum of sequences in a given sample and multiplied by the minimum sum across all samples. Relative abundances were filtered as follows: values below a relative abundance threshold of $0.01 \%$ were not taken into account; taxa with a median relative abundance $<1 \%$ in all groups were not considered for statistical analysis.

(Castillo et al., 2007). In the current study, treatment of RSM was performed prior to ingestion to decrease this adaptation period, but no significant difference was detected in SCFA production among non-processed (CON) and processed RSM (ALK, PECT1, PECT2, and CELL) fermentation during the $48 \mathrm{~h}$ fiber fermentation period in the swine in vitro large intestine model (SLIM). However, when another $24 \mathrm{~h}$ fermentation period was performed after this $48 \mathrm{~h}$ 'fiber adaptation' period, considerable changes were found in the amount of SCFA production in response to different treatments (Long et al. manuscript submitted). Previous studies show that treatments (ALK, PECT1 and PECT2) on RSM did improve the NSP degradation after 4-week adaptation in boilers and pigs (De Vries et al., 2014; Pustjens et al., 2014a). Thus, it is important to know how the microbiota changes during the fiber adaptation period. The current study showed that essentially a $48 \mathrm{~h}$ period was sufficient to increase the pathways, involved in carbohydrate fermentation.
Alpha-diversity significantly decreased after 48 h RSM fermentation, while there were no significant changes between treatments and CON during the fiber adaptation period. This may due to the selection of particular microbes to utilize RSM regardless of the treatment, since RSM was the major nutrient in all cases, compared to the microbial adaptation period $(16 \mathrm{~h}$ SIEMP adaptation), and the different treatments applied only led to changes in cell wall structures in RSM (Pustjens et al., 2014b). In previous studies, it has been shown that RSM increased the relative abundance of Dorea and Lactobacillus in chickens (Chiang et al., 2009; Long et al., 2017). Lachnospira, Coprococcus, Bulleidia, and Shuttleworthia are increased by RSM after three weeks adaptation in pigs (Umu et al., 2018). In the current study, a large number of genera (24 genera) significantly decreased after the fiber fermentation period, and only three and eight genera were considerably increased after adapting to the substrate for 24 and $48 \mathrm{~h}$, respectively. The decreased taxa may not utilize RSM well, while RSM may be beneficial for the increased 
taxa. The significantly increased genera observed in the current study are Dorea, Allisonella, FamilyXIIIUCG_001 (in the order of Clostridiales), Parabacteroides, Mogibacterium, Intestinimonas, Oscillibacter, Sutterella, Citrobacter, RuminococcaceaeUCG_009, and Acidaminococcus. No overlapping genera were observed between the current study and research of Umu et al. (2018), which might be because of a different pig breed. On the other hand, Dorea was found significantly increased by RSM in both the current study and research of Long et al., where six weeks of adaptation period was performed in laying hens (Long et al., 2017). This indicated that Dorea might be a potential RSM degrader.

Disappearance of some low abundance microbes were observed in either processed or non-processed RSM treatment since unweighted UniFrac (only considering presence or absence) was found significantly different $(P=0.02)$, while there was no difference in terms of weighted UniFrac (accounting also for abundance of observed organisms) among the treatments. This indicated that the treatments applied on RSM indeed have an effect on the microbiota composition, but that the short fiber fermentation period used may not yet have led to significant changes. In another study, we showed, however, that weighted UniFrac was observed to be significantly different among the treatments when a single shot of the fibers was introduced after this initial $48 \mathrm{~h}$ (Long et al. manuscript submitted). Zooming in to individual taxa, at the family level, Prevotellaceae significantly increased with CELL. Prevotellaceae is an important family, which is involved in degrading hemicellulose (Emerson and Weimer, 2017). We also observed that genera (e.g., Prevotella 9) from Prevotellaceae significantly positively correlated with SCFA production (Figure 7). To demonstrate the dynamic changes in swine microbiota composition in response to differently pretreated RSM, lumen samples from each time point were analyzed. The most dominant phyla were Firmicutes, Bacteroidetes, and Actinobacteria at $\mathrm{t} 0$, regardless of treatment (Figure 4). This was in line with previous studies (Casellas et al., 2014; Costa et al., 2014; Ramayo-Caldas et al., 2016; Cantu-Jungles et al., 2019), which indicated that core microbes existed. The relative abundance of Bacteroidetes decreased after supplementing with ALK, PECT1, and PECT2, whereas that of Actinobacteria increased (Figure 4). These observations implied that supplementation of ALK, PECT1, and PECT2 stimulated the growth of microbes from phylum Actinobacteria and inhibited the growth of bacteria from phylum Bacteroidetes. Actinobacteria has been reported to efficiently utilize (hemi)cellulose (Munk et al., 2009; Rastogi et al., 2010; Wang et al., 2016). Firmicutes and Proteobacteria were stimulated after supplementing with CELL (Figure 4). Firmicutes is a phylum that consists of a group of dominant butyrate-producing bacteria (Sheridan et al., 2016; La Rosa et al., 2019), which play an important role in carbohydrate degradation. Research showed that the less abundant phylum Proteobacteria enriched gene cohorts of glycolytic enzymes in human gut microbiota (Bradley and Pollard, 2017), which indicated that Proteobacteria might play a similar role in utilizing RSM in the current study. Zooming in to family level, ALK, PECT1, and PECT2 simulated the growth of Atopobiaceae (phylum Actinobacteria); CELL benefited the growth of Lachnospiraceae (phylum Firmicutes). The observations above suggested these microorganisms that were stimulated by these treatments play roles in the fiber degradation. Altogether, this indicated that fiber adaptation period competitively excluded low abundant microbes and created a better ecological niche for the growth of RSMutilization (fiber fermentation) members of the microbiota.

CELL and ALK have larger effect on pig gut microbiota compared to other treatments, since their within-treatment distances between t 0 and 448 were larger than those of CON, PECT1, and PECT2 (Figure 3). Our other study, with the fiber shot after $48 \mathrm{~h}$ adaptation, showed also that more genera were observed to be significantly changed particularly upon feeding CELL and ALK than with PECT1 and PECT2, when compared to CON (Long et al. manuscript submitted). The primary cell wall of RSM contains a backbone of cellulose microfibrils, which are interlinked with xyloglucan via hydrogen bonds forming a stiff network (Carpita and Gibeaut, 1993). Pectins are linked to each other and cross-linked with hemicellulose and cellulose (Broxterman and Schols, 2018). CELL might have relative easy access to target cellulose in RSM, whereas PECT1 and PECT2 could have less opportunity to access pectins, supposedly hindered by cellulose microfibrils. Cell wall structures processed by CELL would lead to utilization of the fibers by microbes, and increase their abundances. Alkaline pretreatment of RSM breaks the alkali-labile bonds, which are known to hinder the complete fermentation of NSP in swine, and improved NSP utilization in feed (Pustjens et al., 2014b). However, also PECT1 and PECT2 affected the swine gut microbiota in these experiments, since gPCA showed that their distances between time point 0 and $48 \mathrm{~h}$ were larger than that of CON. Both gPCA and PCoA are used to classify the dissimilarity between treatments. PCoA might be more often used to classify the dissimilarity between two or more groups, while gPCA can more precisely detect the dissimilarity between two samples based on their distance in the plot. A previous report showed that a cocktail of PECT1 and PECT2 improved degradability of non-starch polysaccharides of RSM in broilers (De Vries et al., 2014). Treatments on RSM, either with carbohydrases or alkaline, did not improve SCFA production during the fiber fermentation period compared to non-processed RSM. This indicated that the processing applied to RSM could not improve their degradability compared to $\mathrm{CON}$, in other words, they might not increase the feed efficiency during this period in vivo. It might be the fiber adaptation period was not enough to express its maximum enzymatic potential (Castillo et al., 2007), although as mentioned earlier within $48 \mathrm{~h}$ the pathways involved in carbohydrate fermentation as increased (Figure 7). Moreover, our own experiments with the fiber shot after $48 \mathrm{~h}$ showed that more pathways related to fiber fermentation were predicted to be upregulated (Long et al. manuscript submitted). PCA plots of Enzyme Classification numbers and pathways also show that samples from t0 clustered together, but separately from samples from t 24 and t48. This also indicated that microbial function between $\mathrm{t} 0$ and later time points was modulated, and geared toward degradation of these new substrates. Previous studies showed that a high-fiber rapeseed diet did not results in a significant increase in SCFA content 
in the chyme of RSM-fed pigs after a 3-week adaptation period (Chen et al., 2018; Umu et al., 2018), which seemed to indicate that the adaptation period was not long enough. Thus, strategies should be considered to shorten the fiber adaptation period, in order to increase feed efficiency.

In conclusion, the current study demonstrated that microbiota composition was significantly affected by both RSM (processed or not) and adaptation time. Unweighted UniFrac showed that microbial community composition was significantly separated between processed RSM and CON, and CELL and ALK in general changed the microbiota composition more than PECT1 and PECT2 did. Carbohydrate metabolism related microbial functions were significantly increased after the fiber fermentation period. However, degradability of the processed RSM was not improved compared to CON during the fiber fermentation period, as assessed by SCFA production, which indicated a relative long adaptation period is needed after a diet change to RSM for swine microbiota. Thus, the significantly different microbes detected between treatments, and the bacteria considerably correlating with SCFA production might be an important finding to design specific strategies to shorten the fiber adaptation period, in order to increase feed efficiency in animals, and particularly in pig production.

\section{DATA AVAILABILITY STATEMENT}

The original contributions presented in the study are publicly available. This data can be found here: https://www.ebi.ac.uk/ena/ data/view/PRJEB36982.

\section{REFERENCES}

Anderson, M. J. (2005). Permutational multivariate analysis of variance. Dep. Statist. Univ. Auckland 26, 32-46.

Bolyen, E., Rideout, J. R., Dillon, M. R., Bokulich, N. A., Abnet, C. C., AlGhalith, G. A., et al. (2019). Reproducible, interactive, scalable and extensible microbiome data science using QIIME 2. Nat. Biotechnol. 37, 852-857. doi: 10.1038/s41587-019-0209-9

Bradley, P. H., and Pollard, K. S. (2017). Proteobacteria explain significant functional variability in the human gut microbiome. Microbiome 5:36. doi: 10.1186/s40168-017-0244-z

Broxterman, S. E., and Schols, H. A. (2018). Interactions between pectin and cellulose in primary plant cell walls. Carbohyd. Polym. 192, 263-272. doi: 10.1016/j.carbpol.2018.03.070

Callahan, B. J., McMurdie, P. J., Rosen, M. J., Han, A. W., Johnson, A. J. A., and Holmes, S. P. (2016). DADA2: high-resolution sample inference from Illumina amplicon data. Nat. Methods 13:581. doi: 10.1038/nmeth.3869

Cantu-Jungles, T. M., do Nascimento, G. E., Zhang, X., Iacomini, M., Cordeiro, L. M., and Hamaker, B. R. (2019). Soluble xyloglucan generates bigger bacterial community shifts than pectic polymers during in vitro fecal fermentation. Carbohyd. Polym. 206, 389-395. doi: 10.1016/j.carbpol.2018.11.011

Carpita, N. C., and Gibeaut, D. M. (1993). Structural models of primary cell walls in flowering plants: consistency of molecular structure with the physical properties of the walls during growth. Plant J. 3, 1-30. doi: 10.1111/j.1365-313x.1993. tb00007.x

Casellas, N. M., Berri, E. M., Fabrellas, J. E., Levenez, F., Lemonnier, G., Denis, C., et al. (2014). Establishment of the swine gut microbiome during early life and further impact on adult health and performance. Intern. Soc. Anim. Genet. Conf. 34.

\section{AUTHOR CONTRIBUTIONS}

$\mathrm{CL}, \mathrm{SV}$, and $\mathrm{KV}$ designed the original study, interpreted the data, and revised the work critically. CL acquired and analyzed the data.

\section{FUNDING}

CL received a Ph.D. scholarship from the China Scholarship Council under the Chinese Government Graduate Student Overseas Study Program (Grant No. 201603250050). The study was funded by the Centre for Healthy Eating \& Food Innovation (HEFI) of Maastricht University campus Venlo and has been made possible with the support of the Dutch Province of Limburg with a grant to HEFI: HEFI-1venk15-25.

\section{ACKNOWLEDGMENTS}

We thank Rob van Dinter, Sanne Verbruggen, and Jessica Verhoeven for technical support.

\section{SUPPLEMENTARY MATERIAL}

The Supplementary Material for this article can be found online at: https://www.frontiersin.org/articles/10.3389/fmicb. 2020.570985/full\#supplementary-material

Castillo, M., Martín-Orúe, S., Anguita, M., Pérez, J., and Gasa, J. (2007). Adaptation of gut microbiota to corn physical structure and different types of dietary fibre. Livest. Sci. 109, 149-152. doi: 10.1016/j.livsci.2007.01.129

Chen, C., Pérez de Nanclares, M., Kurtz, J. F., Trudeau, M. P., Wang, L., Yao, D., et al. (2018). Identification of redox imbalance as a prominent metabolic response elicited by rapeseed feeding in swine metabolome1. J. Anim. Sci. 96, 1757-1768. doi: 10.1093/jas/sky080

Chiang, G., Lu, W., Piao, X., Hu, J., Gong, L., and Thacker, P. (2009). Effects of feeding solid-state fermented rapeseed meal on performance, nutrient digestibility, intestinal ecology and intestinal morphology of broiler chickens. Asian Austr. J. Anim. Sci. 23, 263-271. doi: 10.5713/ajas.2010.90145

Costa, M. O., Chaban, B., Harding, J. C. S., and Hill, J. E. (2014). Characterization of the fecal microbiota of pigs before and after inoculation with "Brachyspira hampsonii”. PLoS One 9:e106399. doi: 10.1371/journal.pone.0106399

de Souza, C. B., Roeselers, G., Troost, F., Jonkers, D., Koenen, M., and Venema, K. (2014). Prebiotic effects of cassava bagasse in TNO's in vitro model of the colon in lean versus obese microbiota. J. Funct. Foods 11, 210-220. doi: 10.1016/j.jff. 2014.09.019

De Vries, S., Pustjens, A., Kabel, M., Kwakkel, R., and Gerrits, W. (2014). Effects of processing technologies and pectolytic enzymes on degradability of nonstarch polysaccharides from rapeseed meal in broilers. Poult. Sci. 93, 589-598. doi: 10.3382/ps.2013-03476

Douglas, G. M., Maffei, V. J., Zaneveld, J., Yurgel, S. N., Brown, J. R., Taylor, C. M., et al. (2019). PICRUSt2: an improved and extensible approach for metagenome inference. bioRxiv [Preprint], doi: 10.1101/672295

Emerson, E. L., and Weimer, P. J. (2017). Fermentation of model hemicelluloses by Prevotella strains and Butyrivibrio fibrisolvens in pure culture and in ruminal enrichment cultures. Appl. Microbiol. Biotechnol. 101, 4269-4278. doi: 10.1007/ s00253-017-8150-7 
Fukuyama, J. (2017). Adaptive gPCA: a method for structured dimensionality reduction. arXiv [Preprint]. Available online at: https://arxiv.org/abs/ 1702.00501\#: \{\}:text=Adaptive $\% 20 \mathrm{gPCA} \% 3 \mathrm{~A} \% 20 \mathrm{~A} \% 20$ method\%20for\% 20structured\%20dimensionality\%20reduction,-Julia\%20Fukuyama\&text= When\%20working\%20with\%20large\%20biological,be\%20tested\%20in\% 20subsequent\%20analyses (accessed December 19, 2019).

Fukuyama, J., Rumker, L., Sankaran, K., Jeganathan, P., Dethlefsen, L., Relman, D. A., et al. (2017). Multidomain analyses of a longitudinal human microbiome intestinal cleanout perturbation experiment. PLoS Comput. Biol. 13:e1005706. doi: 10.1371/journal.pone.01005706

Gibson, G. R., Cummings, J. H., and Macfarlane, G. T. (1988). Use of a three-stage continuous culture system to study the effect of mucin on dissimilatory sulfate reduction and methanogenesis by mixed populations of human gut bacteria. Appl. Environ. Microbiol. 54, 2750-2755. doi: 10.1128/aem.54.11.2750-2755. 1988

Huang, C., Zhang, S., Stein, H. H., Zhao, J., Li, D., and Lai, C. (2018). Effect of inclusion level and adaptation duration on digestible energy and nutrient digestibility in palm kernel meal fed to growing-finishing pigs. Asian Austr. J. Anim. Sci. 31, 395-402. doi: 10.5713/ajas.17.0515

Klindworth, A., Pruesse, E., Schweer, T., Peplies, J., Quast, C., Horn, M., et al. (2013). Evaluation of general 16S ribosomal RNA gene PCR primers for classical and next-generation sequencing-based diversity studies. Nucleic Acids Res. 41:e1. doi: $10.1093 /$ nar/gks808

La Rosa, S. L., Leth, M. L., Michalak, L., Hansen, M. E., Pudlo, N. A., Glowacki, R., et al. (2019). The human gut firmicute Roseburia intestinalis is a primary degrader of dietary $\beta$-mannans. Nat. Commun. 10, 1-14. doi: 10.1038/s41467019-08812-y

Long, C., de Vries, S., and Venema, K. (2020). Polysaccharide source altered ecological network, functional profile, and short-chain fatty acid production in a porcine gut microbiota. Benef. Microb. doi: 10.3920/BM2020.0006

Long, C., Wang, J., Zhang, H., Wu, S., and Qi, G. (2017). Effects of dietary rapeseed meal supplementation on cecal microbiota in laying hens with different flavincontaining monooxygenase 3 genotypes. Poult. Sci. 96, 1748-1758. doi: 10. $3382 /$ ps/pew449

Lozupone, C., and Knight, R. (2005). UniFrac: a new phylogenetic method for comparing microbial communities. Appl. Environ. Microbiol. 71, 8228-8235. doi: 10.1128/aem.71.12.8228-8235.2005

Lozupone, C. A., Hamady, M., Kelley, S. T., and Knight, R. (2007). Quantitative and qualitative beta diversity measures lead to different insights into factors that structure microbial communities. Appl. Environ. Microbiol. 73, 1576-1585. doi: 10.1128/aem.01996-06

Lyu, Z. Q., Huang, C. F., Li, Y. K., Li, P. L., Liu, H., Chen, Y. F., et al. (2018). Adaptation duration for net energy determination of high fiberfibre diets in growing pigs. Anim. Feed Sci. Technol. 241, 15-26. doi: 10.1016/j.anifeedsci. 2018.04.008

Minekus, M., Smeets-Peeters, M., Bernalier, A., Marol-Bonnin, S., Havenaar, R., Marteau, P., et al. (1999). A computer-controlled system to simulate conditions of the large intestine with peristaltic mixing, water absorption and absorption of fermentation products. Appl. Microbiol. Biotechnol. 53, 108-114. doi: 10.1007/ s002530051622

Montoya, C. A., Henare, S. J., Zhu, P., Rutherfurd, S. M., and Moughan, P. J. (2018). Adaptation over time of intestinal fermentation in the growing pig is influenced by the amount of kiwifruit consumed. Br. J. Nutr. 10, 1-43.

Munk, C., Lapidus, A., Copeland, A., Jando, M., Mayilraj, S., Del Rio, T. G., et al. (2009). Complete genome sequence of Stackebrandtia nassauensis type strain (LLR-40K-21 T). Stand. Genom. Sci. 1, 292-299. doi: 10.4056/sigs.47643
Pustjens, A. M. (2013). Fate of Rapeseed Meal Polysaccharides During Digestion in Pigs and Poultry: Effect of Processing and Enzyme Addition. Ph. D thesis, Wageningen University, Wageningen, NL.

Pustjens, A. M., de Vries, S., Bakuwel, M., Gruppen, H., Gerrits, W. J., and Kabel, M. A. (2014a). "Degradability of rapeseed meal polysaccharides in pigs, in Fiberfibre Fermentation in Pigs And Poultry Sense and Nonsense of Its Manipulation: 64, eds W. J. J. Gerrits and M. A. Kabel (Wageningen: Animal Nutrition Group Wageningen University).

Pustjens, A. M., de Vries, S., Bakuwel, M., Gruppen, H., Gerrits, W. J., and Kabel, M. A. (2014b). Unfermented recalcitrant polysaccharide structures from rapeseed (Brassica napus) meal in pigs. Industr. Crops Product. 58, 271-279. doi: 10.1016/j.indcrop.2014.04.036

Pustjens, A. M., de Vries, S., Gerrits, W. J., Kabel, M. A., Schols, H. A., and Gruppen, H. (2012). Residual carbohydrates from in vitro digested processed rapeseed (Brassica napus) meal. J. Agric. Food Chem. 60, 8257-8263. doi: $10.1021 / \mathrm{jf} 301160 \mathrm{~m}$

Ramayo-Caldas, Y., Mach, N., Lepage, P., Levenez, F., Denis, C., Lemonnier, G., et al. (2016). Phylogenetic network analysis applied to pig gut microbiota identifies an ecosystem structure linked with growth traits. ISME J. 10:2973. doi: 10.1038/ismej.2016.77

Rastogi, G., Bhalla, A., Adhikari, A., Bischoff, K. M., Hughes, S. R., Christopher, L. P., et al. (2010). Characterization of Thermostable cellulases produced by Bacillus and Geobacillus strains. Bioresour. Technol. 101, 8798-8806. doi: 10. 1016/j.biortech.2010.06.001

Sáyago-Ayerdi, S. G., Zamora-Gasga, V. M., and Venema, K. (2017). Prebiotic effect of predigested mango peel on gut microbiota assessed in a dynamic in vitro model of the human colon (TIM-2). Food Res. Intern. 118, 89-95. doi: 10.1016/j.foodres.2017.12.024

Segata, N., Izard, J., Waldron, L., Gevers, D., Miropolsky, L., Garrett, W. S., et al. (2011). Metagenomic biomarker discovery and explanation. Genome Biol. 12:R60. doi: 10.1186/gb-2011-12-6-r60

Sheridan, P. O., Martin, J. C., Lawley, T. D., Browne, H. P., Harris, H. M., Bernalier-Donadille, A., et al. (2016). Polysaccharide utilization loci and nutritional specialization in a dominant group of butyrate-producing human colonic Firmicutes. Microb. Genom. 2:e000043. doi: 10.1099/mgen.0.00 0043

Umu, ÖC. O., Fauske, A. K., Åkesson, C. P., de Nanclares, M. P., Sørby, R., Press, C. M., et al. (2018). Gut microbiota profiling in Norwegian weaner pigs reveals potentially beneficial effects of a high-fiberfibre rapeseed diet. PLoS One 13:e0209439. doi: 10.1371/journal.pone.0209439

Wang, C., Dong, D., Wang, H., Müller, K., Qin, Y., Wang, H., et al. (2016). Metagenomic analysis of microbial consortia enriched from compost: new insights into the role of Actinobacteria in lignocellulose decomposition. Biotechnol. Biofuels 9:22. doi: 10.1186/s13068-016-0440-2

Conflict of Interest: The authors declare that the research was conducted in the absence of any commercial or financial relationships that could be construed as a potential conflict of interest.

Copyright (c) 2020 Long, de Vries and Venema. This is an open-access article distributed under the terms of the Creative Commons Attribution License (CC BY). The use, distribution or reproduction in other forums is permitted, provided the original author(s) and the copyright owner(s) are credited and that the original publication in this journal is cited, in accordance with accepted academic practice. No use, distribution or reproduction is permitted which does not comply with these terms. 